OU-HET 705/2011

\title{
$\nu$-Two Higgs Doublet Model and its Collider Phenomenology
}

\author{
${ }^{1}$ Naoyuki Haba, and ${ }^{2}$ Koji Tsumura \\ ${ }^{1}$ Department of Physics, Osaka University, Toyonaka, Osaka 560-0043, Japan \\ ${ }^{2}$ Department of Physics, National Taiwan University, No. 1, Section 4, Roosevelt Rd., \\ Taipei, Taiwan
}

\begin{abstract}
Smallness of neutrino masses can be explained by introducing a tiny vacuum expectation value of an extra-Higgs doublet which couples to righthanded neutrinos $\left(N_{R}\right)$. This situation is naturally realized in $\nu$-Two Higgs Doublet Model ( $\nu$ THDM), where a TeV-scale seesaw mechanism can work well. We investigate observable phenomenology of $\nu$ THDM at LHC and ILC experiments. Charged Higgs boson $\left(H^{ \pm}\right)$in $\nu$ THDM is almost originated from the extra-Higgs doublet and its coupling strength to neutrinos are not small. Then this model induces rich phenomenology at the LHC, for example, when $m_{H^{ \pm}}<M_{N}$, observable charged tracks can be induced from long lived charged Higgs. On the other hand, when $m_{H^{ \pm}}>M_{N}$, right-handed neutrinos can be long-lived, and secondary vertices may be tagged at the LHC. The $\nu$ THDM also predicts observable lepton number violating process at the ILC.
\end{abstract}




\section{Introduction}

Recent progress in neutrino oscillation experiments gradually reveal a structure of lepton sector [1, 2]. However, from a theoretical point of view, smallness of neutrino masses is still a mystery in the standard model (SM), and it is one of the most important clues to find new physics beyond the SM. It is known that the seesaw mechanism naturally realizes tiny masses of active neutrinos through heavy particles coupled with left-handed neutrinos[3]. However, those heavy particles are almost decoupled in the low-energy effective theory, few observations may be expected in collider experiments. Then, a possibility have been discussed to reduce seesaw scales to be $\mathrm{TeV}$ [4, 目], where effects of TeV scale right-handed neutrinos might be observed in collider experiments such as Large Hadron Collider (LHC) and International Linear Collider (ILC). However, they must introduce fine-tunings in order to obtain both tiny neutrino masses and a detectable left-right neutrino mixing through which they insist experimental evidences can be discovered [6]. Other right-handed neutrino production processes in extended models by e.g., $Z^{\prime}$ exchange [7, 8] or Higgs/Higgsino decay [9] have been also pointed out.

Here let us take a different approach. We remind that Dirac masses of fermions are proportional to their Yukawa couplings as well as a vacuum expectation value (VEV) of the relevant Higgs field. Hence, the smallness of masses might be due to not a small Yukawa coupling but a small VEV of the Higgs field. Such a situation is indeed realized in some twoHiggs-doublet model (THDM). For example, in Type-II THDM, the mass hierarchy between up-type quark and down-type quark can be explained by the ratio $(\tan \beta)$ of VEVs, and when $\tan \beta \sim 40$, Yukawa couplings of top- and bottom-quark are the same scale of order of unity [10]. Similarly, there is a possibility that smallness of neutrino masses comparing to those of quarks and charged leptons is originating from an extra Higgs doublet with the tiny VEV. This idea is that neutrino masses are much smaller than other fermions because the origin of them comes from different VEV of different Higgs doublet, and then we do not need extremely tiny neutrino Yukawa coupling constants. This kind of model has been considered in Refs. 11, 12, 13, 14, 15, 16. We call the model $\nu$ THDM in this paper, where TeV-scale seesaw can work, and investigate detective possibilities of the model in collider experiments. Especially, in models in Refs. [11, 16], tiny Majorana neutrino masses are obtained through a TeV scale Type-I seesaw mechanism without requiring tiny Yukawa couplings. The fact that neutrino Yukawa couplings in the $\nu$ THDM are not so small, which also makes low energy thermal leptogenesis work 17.

As for extending a Higgs sector, there are constraints in general, for example, consistency of electroweak precision data and absence of large flavor changing neutral currents (FCNCs) 19, 21. In this model, both two constraints are satisfied since the extra scalar doublet only has

* It is pointed out that the second Higgs doublet heavier than SM-like Higgs boson can be consistent with precision electroweak data 18 . 
a Yukawa interaction with lepton doublets and right-handed neutrinosi, and their masses are heavy enough to suppress FCNCs although its VEV is of order $0.1 \mathrm{MeV}$. Decay processes of charged Higgs boson can induce important phenomenology in our model, since it is almost originated from the extra-Higgs doublet and its couplings to neutrinos are not small. Note that the decay of the charged Higgs boson to quarks are strongly suppressed due to absence of direct interactions among them, which is a large difference from other THDMs. When $m_{H^{ \pm}}<M_{N}$, charged tracks of long lived charged Higgs boson can be found at the LHC. On the other hand, when $m_{H^{ \pm}}>M_{N}$, secondary vertices can be tagged at the LHC, since righthanded neutrinos can be long-lived. In our setup, light neutrinos are considered as Majorana particles, so that lepton number violating processes could also be detectable at ILC. We will show it especially by analyzing a process of $e^{-} e^{-} \rightarrow H^{-} H^{-}$. Anyhow, we stress again that characteristic signals of the $\nu$ THDM could be detected at LHC and ILC experiments, and investigating them is our purpose of this paper.

\section{Setup}

At first, in this section, let us show a structure of the $\nu$ THDM with $N_{R}$ and an example of its UV theory.

\section{1 $\nu$ THDM with right-handed Majorana masses}

The $\nu$ THDM is originally suggested in Ref. [11]. In a framework of this model, Majorana neutrinos were studied in Refs.[11, 13 and Dirac neutrinos in Ref. 12, 14. In the model, one additional Higgs doublet $\Phi_{\nu}$, which gives only neutrino Dirac masses, is introduced besides the SM Higgs doublet $\Phi$ as

$$
\Phi_{\nu}=\left(\begin{array}{c}
\phi_{\nu}^{+} \\
\phi_{\nu}^{0}
\end{array}\right), \Phi=\left(\begin{array}{c}
\phi^{+} \\
\phi^{0}
\end{array}\right) .
$$

We introduce a discrete $Z_{2}$-parity which distinguishes $\Phi_{\nu}$ from $\Phi$, and the $Z_{2}$-charges (and also lepton number) are assigned as the following table.

\begin{tabular}{|l|c|c|}
\hline fields & $Z_{2}$-parity & lepton number \\
\hline \hline SM Higgs doublet, $\Phi$ & + & 0 \\
\hline new Higgs doublet, $\Phi_{\nu}$ & - & 0 \\
\hline right-handed neutrinos, $N_{R}$ & - & 1 \\
\hline others & + & \pm 1: leptons, 0: quarks \\
\hline
\end{tabular}

$\dagger$ This is a kind of a "leptophilic Higgs boson" which could explain PAMERA and ATIC results 22. 
Under the discrete symmetry, Yukawa interactions and Majorana mass terms of right-handed neutrinos are given by

$$
-\mathcal{L}_{\text {Yukawa }}=\bar{Q} Y_{u} U_{R} \tilde{\Phi}+\bar{Q} Y_{d} D_{R} \Phi+\bar{L} Y_{e} E_{R} \Phi+\bar{L} Y_{\nu} N_{R} \tilde{\Phi}_{\nu}+\frac{1}{2} \overline{N_{R}{ }^{c}} M_{N} N_{R}+\text { H.c. }
$$

where $\tilde{\Phi}=i \sigma_{2} \Phi^{*}$, and we omit a generation index. We here take the real diagonal bases for charged lepton and right-handed neutrino mass matrices without loss of generality. An extra scalar doublet $\Phi_{\nu}$ only couples with $N_{R}$ by the $Z_{2}$-parity, so that FCNCs are forbidden at tree level. Quark and charged lepton sectors are the same as Type-I THDM [19], but notice that this $\nu$ THDM is quite different from conventional Type-I, II, X, Y THDMs [20] due to the tiny VEV.

Now let us concentrate on the neutrino sector. From Eq. (2.2), the general mass matrix for Majorana neutrino is given by

$$
-\frac{1}{2}\left(\begin{array}{ll}
\overline{\nu_{L}^{\prime c}} & \overline{N_{R}^{\prime}}
\end{array}\right)\left(\begin{array}{cc}
0 & \frac{v_{\nu}}{\sqrt{2}} Y_{\nu}^{*} \\
\frac{v_{\nu}}{\sqrt{2}} Y_{\nu}^{\dagger} & M_{N}
\end{array}\right)\left(\begin{array}{c}
\nu_{L}^{\prime} \\
N_{R}^{\prime c}
\end{array}\right)+\text { H.c. }
$$

where we denote $\left\langle\phi_{\nu}^{0}\right\rangle=v_{\nu} / \sqrt{2}$. If $\mathcal{O}\left(Y_{\nu} v_{\nu} / M_{N}\right) \ll 1$, the matrix can be approximately block diagonalized as

$$
\left(\begin{array}{c}
\nu_{L}^{\prime} \\
N_{R}^{\prime c}
\end{array}\right)=\left(\begin{array}{cc}
\mathbf{1} & +\frac{v_{\nu}}{\sqrt{2}} Y_{\nu} M_{N}^{-1} \\
-\frac{v_{\nu}}{\sqrt{2}} M_{N}^{-1} Y_{\nu}^{\dagger} & \mathbf{1}
\end{array}\right)\left(\begin{array}{c}
U_{\mathrm{MNS} \nu_{L}} \\
N_{R}{ }^{c}
\end{array}\right) .
$$

where $U_{\text {MNS }}$ is the neutrino mixing matrix. Mass eigenstates for light neutrinos are determined by diagonalization of $3 \times 3$ block as

$$
m_{\nu}=-\frac{v_{\nu}^{2}}{2} Y_{\nu} M_{N}^{-1} Y_{\nu}^{T}=U_{\mathrm{MNS}} m_{\nu}^{\text {diag }} U_{\mathrm{MNS}}^{T},
$$

where $m_{\nu}^{\text {diag }}$ is real diagonal. In order to realize tiny neutrino masses, parameters can be chosen as i) small $Y_{\nu}$, ii) tiny $v_{\nu}$, or iii) huge $M_{N}$. Here the $\nu$ THDM focuses on the second possibility which may give interesting Higgs and $N_{R}$ phenomenology due to the relatively large neutrino Yukawa coupling and the lighter right-handed neutrinos $\left(M_{N}\right.$ is supposed to be below the $\mathrm{TeV}$ scale). Actually, radiative effects also induce Majorana neutrino masses, which are estimated later.

The Higgs potential of the $\nu$ THDM is given by

$$
\begin{aligned}
V^{\mathrm{THDM}}= & m_{\Phi}^{2} \Phi^{\dagger} \Phi+m_{\Phi_{\nu}}^{2} \Phi_{\nu}^{\dagger} \Phi_{\nu}-m_{3}^{2}\left(\Phi^{\dagger} \Phi_{\nu}+\Phi_{\nu}^{\dagger} \Phi\right)+\frac{\lambda_{1}}{2}\left(\Phi^{\dagger} \Phi\right)^{2}+\frac{\lambda_{2}}{2}\left(\Phi_{\nu}^{\dagger} \Phi_{\nu}\right)^{2} \\
& +\lambda_{3}\left(\Phi^{\dagger} \Phi\right)\left(\Phi_{\nu}^{\dagger} \Phi_{\nu}\right)+\lambda_{4}\left(\Phi^{\dagger} \Phi_{\nu}\right)\left(\Phi_{\nu}^{\dagger} \Phi\right)+\frac{\lambda_{5}}{2}\left[\left(\Phi^{\dagger} \Phi_{\nu}\right)^{2}+\left(\Phi_{\nu}^{\dagger} \Phi\right)^{2}\right]
\end{aligned}
$$


The $Z_{2}$-symmetry is softly broken by $m_{3}^{2}$. Taking a parameter set,

$$
m_{\Phi}^{2}<0, \quad m_{\Phi_{\nu}}^{2}>0, \quad\left|m_{3}^{2}\right| \ll m_{\Phi_{\nu}}^{2},
$$

and denoting $\left\langle\phi^{0}\right\rangle=v / \sqrt{2}$, we can obtain the VEV hierarchy of Higgs doublets,

$$
v^{2} \simeq \frac{-2 m_{\Phi}^{2}}{\lambda_{1}}, \quad v_{\nu} \simeq \frac{-m_{3}^{2} v}{m_{\Phi_{\nu}}^{2}+\left(\lambda_{3}+\lambda_{4}+\lambda_{5}\right) v^{2} / 2} .
$$

When we take values of parameters as $m_{\Phi} \sim 100 \mathrm{GeV}, m_{\Phi_{\nu}} \sim 1 \mathrm{TeV}$, and $\left|m_{3}^{2}\right| \sim 10 \mathrm{GeV}^{2}$, we can obtain $v_{\nu} \sim 1 \mathrm{MeV}$. The smallness of $\left|m_{3}^{2}\right|$ is guaranteed by the "softly-broken" $Z_{2^{-}}$ symmetry. This VEV hierarchy is preserved after estimating a one-loop effective potential as long as $m_{\Phi_{\nu}}$ is larger than weak scale, since modifications of stationary conditions are negligible.

For a large $\tan \beta=v / v_{\nu}(\gg 1)$ limit we are interested in, five physical Higgs states and those masses are given by

$$
\begin{array}{rlrl}
h & \simeq \operatorname{Re}\left[\phi^{0}\right], & m_{h}^{2} \simeq 2 \lambda_{1} v^{2}, \\
H \simeq \operatorname{Re}\left[\phi_{\nu}^{0}\right], & m_{H}^{2} \simeq m_{\Phi_{\nu}}^{2}+\frac{1}{2}\left(\lambda_{3}+\lambda_{4}+\lambda_{5}\right) v^{2}, \\
A \simeq \operatorname{Im}\left[\phi_{\nu}^{0}\right], & m_{A}^{2} \simeq m_{\Phi_{\nu}}^{2}+\frac{1}{2}\left(\lambda_{3}+\lambda_{4}-\lambda_{5}\right) v^{2}, \\
H^{ \pm} \simeq\left[\phi_{\nu}^{ \pm}\right], & m_{H^{ \pm}}^{2} \simeq m_{\Phi_{\nu}}^{2}+\frac{1}{2} \lambda_{3} v^{2},
\end{array}
$$

respectively, where we omit negligible corrections of $\mathcal{O}\left(v_{\nu}^{2}\right)$ and $\mathcal{O}\left(m_{3}^{2}\right)$. Notice that $\tan \beta$ is extremely large, so that the SM-like Higgs boson $h$ is almost originated from $\Phi$, while other physical Higgs particles, $H^{ \pm}, H, A$, are almost originated from $\Phi_{\nu}$. Since $\Phi_{\nu}$ has Yukawa couplings only with right-handed neutrinos and lepton doublets, remarkable phenomenology can be expected which are not observed in other THDMs. As early works, lepton flavor violation (LFV) processes and oblique corrections in $\nu$ THDM are estimated in Ref. [11], and charged Higgs boson phenomenology in collider experiments are discussed in Refs. 14, 15] f.

Now we are in a position to estimate the neutrino mass from one-loop radiative corrections. There are one-loop diagrams which induce Majorana neutrino masses [13]. The effects exist when $\lambda_{5} \neq 0$, and the dimension five operator is induced. It is estimated as

$$
\begin{aligned}
\left(m_{\nu}^{\text {loop }}\right)_{i j} & =-\sum_{k} \frac{\left(Y_{\nu}\right)_{i k}\left(Y_{\nu}\right)_{j k} M_{R k}}{16 \pi^{2}}\left[\frac{m_{H}^{2}}{m_{H}^{2}-M_{R k}^{2}} \ln \frac{m_{H}^{2}}{M_{R k}^{2}}-\frac{m_{A}^{2}}{m_{A}^{2}-M_{R k}^{2}} \ln \frac{m_{A}^{2}}{M_{R k}^{2}}\right] \\
& \simeq-\frac{\lambda_{5} v^{2}}{8 \pi^{2}} \sum_{k} \frac{\left(Y_{\nu}\right)_{i k}\left(Y_{\nu}\right)_{j k} M_{R k}}{m_{0}^{2}-M_{R k}^{2}}\left[1-\frac{M_{R k}^{2}}{m_{0}^{2}-M_{R k}^{2}} \ln \frac{m_{0}^{2}}{M_{R k}^{2}}\right],
\end{aligned}
$$

$\ddagger$ The model[14, 15] deals with Dirac neutrino version in $\nu$ THDM, and phenomenology of charged Higgs has a similar region in part. 

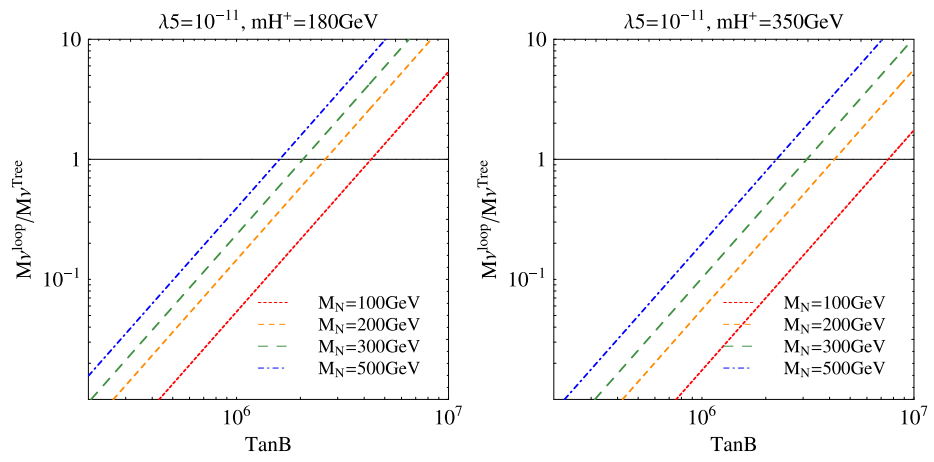

Figure 1: The ratio of the tree level and the loop level contributions to the neutrino mass.

where $m_{H}^{2}-m_{A}^{2}=\lambda_{5} v^{2} \ll m_{0}^{2}=\left(m_{H}^{2}+m_{A}^{2}\right) / 2$. If the masses of Higgs bosons (except for $h$ ) and right-handed neutrinos are $\mathcal{O}(1) \mathrm{TeV}$, light neutrino mass scale of order $\mathcal{O}(0.1) \mathrm{eV}$ is induced with $\lambda_{5} \sim 10^{-4}$ for $Y_{\nu} \sim 10^{-3}$. Whether tree-level effect is larger than loop-level effect or not is determined by the magnitude of $\lambda_{5}$. Roughly speaking, when $\lambda_{5} v^{2} /(4 \pi)^{2} \gtrsim v_{\nu}^{2}$, radiatively induced mass would be dominated. If we consider the Type-I seesaw dominance, $v_{\nu}$ would be a valuable parameter. By fixing the light neutrino mass and $M_{N}$, neutrino data can be fit for wide range of parameter space in $v_{\nu}$ with a change the magnitudes of $Y_{\nu}$. And, since the magnitude of $v_{\nu}$ is determined by $m_{3}^{2}$, it is the essential parameter in $\nu$ THDM. We show the ratio of contributions to the light Majorana neutrino mass from the tree level and radiative seesaws as a function of $\tan \beta$ in FIG. [. Two models, vanishing $v_{\nu}[13$ and nonvanishing $v_{\nu}$ [1], are connected by the magnitude of $v_{\nu}$. The former model has exact $Z_{2}$-parity with $m_{3}^{2}=0$, where right-handed neutrino becomes dark matter and masses of light active neutrinos are fully induced by the radiative corrections as discussed above.

\subsection{An example of UV theory of $\nu$ THDM}

Next, let us show an example of UV theory 16 of the $\nu$ THDM. This model is constructed by introducing one gauge singlet scalar field $S$, which has a lepton number, and $Z_{3}$-charges are shown as the following table. Under the discrete symmetry, Yukawa interactions are given by

\begin{tabular}{|l|c|c|}
\hline fields & $Z_{3}$-charge & lepton number \\
\hline \hline SM Higgs doublet, $\Phi$ & 1 & 0 \\
\hline new Higgs doublet, $\Phi_{\nu}$ & $\omega^{2}$ & 0 \\
\hline new scalar singlet, $S$ & $\omega$ & -2 \\
\hline right-handed neutrinos, $N_{R}$ & $\omega$ & 1 \\
\hline others & 1 & \pm 1 : leptons, 0: quarks \\
\hline
\end{tabular}




$$
-\mathcal{L}_{\text {Yukawa }}=\bar{Q} Y_{u} U_{R} \tilde{\Phi}+\bar{Q} Y_{d} D_{R} \Phi+\bar{L} Y_{e} E_{R} \Phi+\bar{L} Y_{\nu} N_{R} \tilde{\Phi}_{\nu}+\frac{1}{2} S Y_{N} \overline{N_{R}} N_{R}+\text { H.c. }
$$

Notice that the effective Majorana masses are induced in the $\nu$ THDM as

$$
M_{R}=Y_{N}\langle S\rangle .
$$

The Higgs potential can be written as

$$
\begin{gathered}
V=m_{\Phi}^{2}|\Phi|^{2}+m_{\Phi_{\nu}}^{2}\left|\Phi_{\nu}\right|^{2}-m_{S}^{2}|S|^{2}+\frac{\lambda_{1}}{2}|\Phi|^{4}+\frac{\lambda_{2}}{2}\left|\Phi_{\nu}\right|^{4}+\lambda_{3}|\Phi|^{2}\left|\Phi_{\nu}\right|^{2}+\lambda_{4}\left|\Phi^{\dagger} \Phi_{\nu}\right|^{2} \\
+\lambda_{S}|S|^{4}+\lambda_{\Phi}|S|^{2}|\Phi|^{2}+\lambda_{\Phi_{\nu}}|S|^{2}\left|\Phi_{\nu}\right|^{2}+\left[-\lambda S^{3}-\kappa S \Phi^{\dagger} \Phi_{\nu}+\text { H.c. }\right] .
\end{gathered}
$$

$Z_{3}$-symmetry forbids dimension four operators, $\left(\Phi^{\dagger} \Phi_{\nu}\right)^{2}, \Phi^{\dagger} \Phi_{\nu}|\Phi|^{2}, \Phi^{\dagger} \Phi_{\nu}\left|\Phi_{\nu}\right|^{2}, S^{4}, S^{2}|S|^{2}$, $S^{2}|\Phi|^{2}, S^{2}\left|\Phi_{\nu}\right|^{2}$, and dimension two or three operators, $\Phi^{\dagger} \Phi_{\nu}, S|\Phi|^{2}, S\left|\Phi_{\nu}\right|^{2}$. Although there might be introduced small soft breaking terms such as $m_{3}^{\prime 2} \Phi^{\dagger} \Phi_{\nu}$ to avoid domain wall problem, we omit them here, for simplicity. It has been shown that, with $\kappa \sim 1 \mathrm{MeV}$, the desirable hierarchy of VEVs

$$
v_{s} \equiv\langle S\rangle \sim 1 \mathrm{TeV}, \quad v \sim 100 \mathrm{GeV}, \quad v_{\nu} \sim 1 \mathrm{MeV},
$$

and neutrino mass $m_{\nu} \sim \frac{Y_{\nu}^{2} v_{\nu}^{2}}{M_{R}}$ can be realized [16]. This is so-called Type-I seesaw mechanism in a TeV scale, when coefficients $Y_{\nu}$ and $Y_{N}$ are assumed to be of order one. The masses of scalar and pseudo-scalar mostly from VEV of $S$ are given by

$$
m_{H_{S}}^{2}=m_{S}^{2}+2 \lambda_{S} v_{s}^{2}, \quad m_{A_{S}}^{2}=9 \lambda v_{s},
$$

where we assume the $\mathrm{CP}$ invariant Higgs sector. For parameter region with $v_{s} \gg 1 \mathrm{TeV}$, both scalar and pseudo-scalar are heavier than other particles. After integrating out $S$, thanks to the $Z_{3}$-symmetry, the model ends up with an effectively $\nu$ THDM with approximated $Z_{2^{-}}$ symmetry, $\Phi \rightarrow \Phi, \Phi_{\nu} \rightarrow-\Phi_{\nu}$. Comparing to the $\nu$ THDM, the value of $m_{3}^{2}$, which is a soft $Z_{2}$-symmetry breaking term, is expected to be $\kappa v_{s} . \lambda_{5}$ is induced by integrating out $S$, which is estimated as $\mathcal{O}\left(\kappa^{2} / m_{S}^{2}\right) \sim 10^{-12}$.

As for the neutrino mass induced from one-loop diagram, UV theory induces small $\lambda_{5} \sim$ $10^{-12}$ as shown above, then the radiatively induced neutrino mass from one-loop diagram is estimated as $\lambda_{5} v^{2} /(4 \pi)^{2} M \sim 10^{-4} \mathrm{eV}$. This can be negligible comparing to light neutrino mass which is induced from tree level Type-I seesaw mechanism. The tree level neutrino mass is

$$
m_{\nu}^{\text {tree }} \sim \frac{y_{\nu}^{2} v_{\nu}^{2}}{M} \sim \frac{y_{\nu}^{2} \kappa^{2} v^{2}}{v_{s}^{2} M},
$$

where we input $v_{\nu} \sim \frac{k v}{v_{s}}$. On the other hand, one-loop induced neutrino mass is estimated as

$$
m_{\nu}^{\text {loop }} \sim \frac{\lambda_{5} y_{\nu}^{2}}{(4 \pi)^{2}} \frac{v^{2}}{M} \sim \frac{y_{\nu}^{2}}{(4 \pi)^{2}} \frac{\kappa^{2} v^{2}}{M^{2} M} .
$$


Putting $M \sim v_{s}$,

$$
\frac{m_{\nu}^{\text {loop }}}{m_{\nu}^{\text {tree }}} \sim \frac{1}{(4 \pi)^{2}}
$$

which shows loop induced neutrino mass is always smaller than tree level mass if UV theory is the model of Ref. [16].

\section{$3 \quad$ Phenomenology in $\nu$ THDM with $N_{R}$}

In this section, we study phenomenology in the $\nu$ THDM with $N_{R}$, which makes a low energy seesaw mechanism work. At first, we will briefly show experimental constraints of the $\nu$ THDM. And next, we will show observable collider phenomenology in LHC and ILC experiments.

\subsection{Experimental constraints}

Let us summarize experimental constraints on the model, and the first is LFV. The muon LFV is precisely measured, and MEGA experiment gives a strong upper limit as $\mathcal{B}_{\mu \rightarrow e \gamma}<$ $1.2 \times 10^{-11}[23$. The branching fraction of radiative LFV decay due to neutrinophilic charged Higgs boson is calculated as 11

$$
\mathcal{B}_{\mu \rightarrow e \gamma}=\frac{3 \alpha_{\mathrm{EM}}}{2 \pi}\left|\frac{v^{2}}{2 m_{H^{ \pm}}^{2}}\left(Y_{\nu}\right)_{e N}\left(Y_{\nu}\right)_{\mu N}^{*} F_{2}\left(\frac{M_{N}^{2}}{m_{H^{ \pm}}^{2}}\right)\right|^{2},
$$

where

$$
F_{2}(t)=\frac{1}{12(1-t)^{4}}\left(1-6 t+3 t^{2}+2 t^{3}-6 t^{2} \ln t\right) .
$$

Assuming masses of charged Higgs boson and right-handed neutrino to be TeV scale, $Y_{\nu} \lesssim 0.1$ should be satisfied. When the neutrino mass dominantly induced by Type-I seesaw mechanism, the neutrino Yukawa coupling matrix can be expressed as [24]

$$
Y_{\nu} \simeq i \frac{\sqrt{2}}{v_{\nu}} U_{\mathrm{MNS}} \widehat{m}_{\nu}^{1 / 2} R \widehat{M}_{N}^{1 / 2}=i \frac{\sqrt{2}}{v} U_{\mathrm{MNS}} \widehat{m}_{\nu}^{1 / 2} R \widehat{M}_{N}^{1 / 2} \tan \beta,
$$

where $R$ is a complex orthogonal matrix. Small $v_{\nu}$, equivalently large $\tan \beta$, is constrained by $\mu \rightarrow e \gamma$ results. However, we note that small $v_{\nu}$ is not excluded, because the one-loop contribution to neutrino mass is dominated in this case (see FIG. 1), and hence Eq.(3.24) is no longer valid.

Next is about $\rho$-parameter, and it often gives severe constraints for models of beyond the SM[19]. Since $\rho$-parameter characterizes breaking of custodial $S U(2)$ symmetry, we here

simply requires mass degeneracy of extra Higgs bosons, i.e., $m_{A} \simeq m_{H^{ \pm}}$. This assumption is automatically satisfied when $m_{\Phi_{\nu}}^{2} \gtrsim v^{2}$. 
The last constraint is neutrinoless double beta decay. As in the SM, masses of light Majorana neutrinos are the source of neutrinoless double beta decay 25]. In the $\nu$ THDM, charged Higgs bosons are quarkophobic, so that it cannot affect to neutrinoless double beta decay.

\subsection{LHC phenomenology}

Now let us investigate LHC physics of the $\nu$ THDM. For this purpose, it is good for us to focus on decays of charged Higgs bosons and right-handed neutrinos. It is because we can expect the charged Higgs boson production at the LHC, and its decay processes can be observable[26]. The charged Higgs boson in the $\nu$ THDM is almost composed by $\Phi_{\nu}$ whose couplings to quarks are negligibly small. Therefore, different phenomenology from other THDMs are expected to be observed in accelerator experiments especially in the processes related with charged Higgs particle and right-handed neutrinos which have significant Yukawa interactions. Here let us summarize decays of charged Higgs bosons and also right-handed neutrinos, depending on mass spectrum.

\subsection{1 $m_{H^{ \pm}}<M_{N}$}

For $m_{H^{ \pm}}<M_{N}, N_{R}$ decays into leptons and on-shell scalar bosons via the neutrino Yukawa interaction as

$$
\begin{aligned}
\Gamma\left(N_{R a} \rightarrow \ell^{+} H^{-}\right) & =\frac{\left|\left(Y_{\nu}\right)_{\ell a}\right|^{2} m_{H^{ \pm}}^{2}}{16 \pi M_{a}}\left(1-\frac{m_{H^{ \pm}}^{2}}{M_{a}^{2}}\right)^{2}, \\
\Gamma\left(N_{R a} \rightarrow \nu_{i} \phi_{\nu}^{0}\right) & =\frac{\left|\left(U_{\mathrm{MNS}}^{\dagger} Y_{\nu}\right)_{i a}\right|^{2} m_{\phi_{\nu}^{0}}^{2}}{16 \pi M_{a}}\left(1-\frac{m_{\phi_{\nu}^{0}}^{2}}{M_{a}^{2}}\right)^{2},
\end{aligned}
$$

where $\phi_{\nu}^{0}=H, A$, and lepton masses are neglected. However, it would be difficult to produce $N_{R}$ at the LHC even when their masses are below $1 \mathrm{TeV}$, because right-handed neutrinos are originally gauge singlet, and their weak interactions is suppressed by $Y_{\nu} v_{\nu} / M_{N}$.

On the other hand, charged Higgs bosons can easily be produced at the LHC via the scalar pair creation process $q \bar{q} \rightarrow \gamma^{*}\left(Z^{*}\right) \rightarrow H^{+} H^{-}$[26]. For large $\tan \beta=v / v_{\nu}$, the main decay modes of charged Higgs boson would be light neutrinos and charged leptons via the Yukawa interaction. Because of small mixing between light- and heavy- neutrinos, the decay width is strongly suppressed as

$$
\Gamma\left(H^{+} \rightarrow \ell^{+} \nu_{L i}\right)=\frac{\left|\left(Y_{\nu} M_{N}^{-1} Y_{\nu}^{T} U_{\mathrm{MNS}}^{*}\right) \ell_{i}\right|^{2} m_{H^{ \pm}}}{32 \sqrt{2} \pi G_{F} \tan ^{2} \beta} .
$$

If $\tan \beta$ is not so large, small mixing in scalar sector becomes important. In this case, charged Higgs boson can also decay into quarks. 

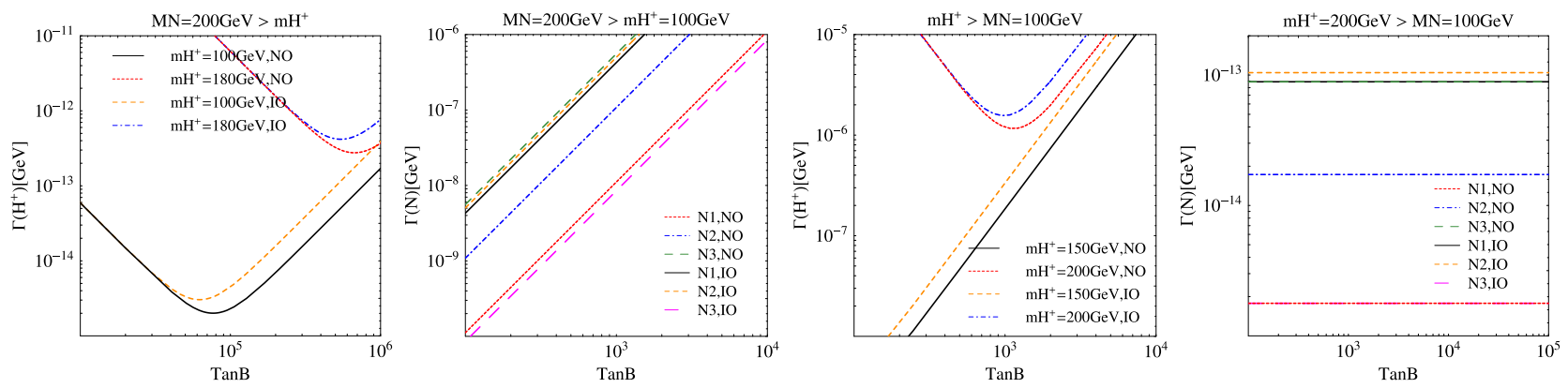

Figure 2: Decay widths for the charged Higgs boson and right-handed neutrinos are shown, where $R=\mathbf{1}$ and $M_{N} \propto \mathbf{1}$ are taken.
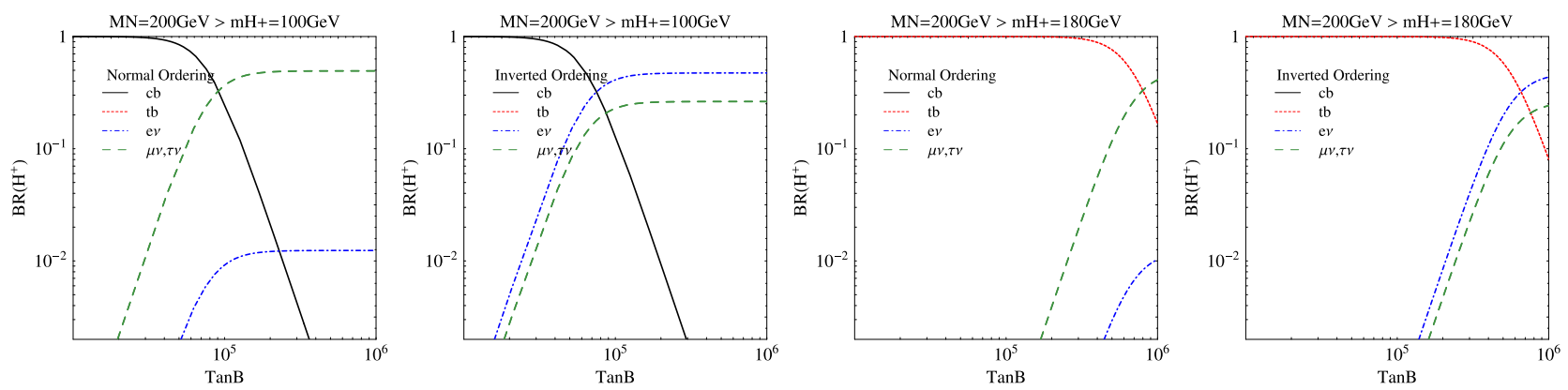

Figure 3: Decay branching ratios of the charged Higgs boson are given for $m_{H^{ \pm}}<M_{N}$, where $R=\mathbf{1}$ and $M_{N} \propto \mathbf{1}$ are taken.

In the first panel of FIG. 2, we show the total decay width of charged Higgs boson. We here take the charged Higgs boson mass to be $100 \mathrm{GeV}$ and $180 \mathrm{GeV}$, and $R=\mathbf{1}$ and $M_{N} \propto \mathbf{1}$ are assumed. Mass spectrum of light neutrinos is considered for both normal mass ordering and inverted mass ordering case. It must be stressed that the decay length of charged Higgs bosons is found to be longer, so that long lived charged Higgs boson can be found at the LHC. If the charged Higgs boson is heavier than the top-quark, charged Higgs boson can also decay into $t b$ pairs. In this case, the lifetime of charged Higgs bosons is shorter by factor of $m_{c}^{2} / m_{t}^{2}$. In the second panel, we also show the total decay width of right-handed Majorana neutrinos. Heavy neutrinos instantaneously decay into leptons and scalars. Outgoing charged leptons can be tagged if right-handed neutrinos are produced.

In FIG. 3, decay branching ratios of charged Higgs boson are shown, where $m_{H^{ \pm}}=100$ $\mathrm{GeV}$ (first and second) and $180 \mathrm{GeV}$ (third and fourth) are taken, and neutrino mass spectrum are assumed to be normal mass ordering and inverted mass ordering. For lower tan $\beta$ region, charged Higgs bosons mainly decay into quarks, while for larger $\tan \beta$ region they do into leptons. In large $\tan \beta$ region, main leptonic decay channels are different in the normal ordering 
case and in the inverted ordering cases.

\subsection{2 $m_{H^{ \pm}}>M_{N}$}

For $m_{H^{ \pm}}>M_{N}$, charged Higgs bosons cannot be long-lived. The charged Higgs bosons $\left(H^{+}\right)$rapidly decay into charged leptons $\left(\ell^{+}\right)$and on-shell right-handed neutrinos $N_{R}$ via the neutrino Yukawa interaction as

$$
\Gamma\left(H^{+} \rightarrow \ell^{+} N_{R a}\right)=\frac{\left|\left(Y_{\nu}\right)_{\ell a}\right|^{2} m_{H^{ \pm}}}{16 \pi}\left(1-\frac{M_{a}^{2}}{m_{H^{ \pm}}^{2}}\right)^{2} .
$$

As we mentioned before, charged Higgs boson can easily be produced at the LHC, and then right-handed neutrinos can be created from their decays. Decays of $H$ and $A$ are also used to generate right-handed neutrinos. Subsequently, $N_{R}$ decays into leptons and gauge bosons as

$$
\begin{aligned}
\Gamma\left(N_{R a} \rightarrow \ell^{+} W^{-}\right) & =\frac{\left|\left(Y_{\nu}\right)_{\ell a}\right|^{2} M_{a}}{32 \pi \tan ^{2} \beta}\left(1-\frac{m_{W}^{2}}{M_{a}^{2}}\right)\left(1+\frac{2 m_{W}^{2}}{M_{a}^{2}}\right), \\
\Gamma\left(N_{R a} \rightarrow \overline{\nu_{L}} Z\right) & =\frac{\left|\left(U_{\mathrm{MNS}}^{\dagger} Y_{\nu}\right)_{i a}\right|^{2} M_{a}}{64 \pi \tan ^{2} \beta}\left(1-\frac{m_{Z}^{2}}{M_{a}^{2}}\right)\left(1+\frac{2 m_{Z}^{2}}{M_{a}^{2}}\right),
\end{aligned}
$$

and also into neutrinos and scalar bosons $(h, H, A)$ if kinematically allowed. Due to the strong suppression, i.e., $1 / \tan ^{2} \beta$, right-handed neutrinos may be long-lived. It seems difficult to consider the dark matter candidate, since the lifetime of right-handed neutrinos cannot be large compared to that of universe.

In the third panel of FIG. 2, the total decay width of charged Higgs bosons is shown. For smaller values of $\tan \beta$, charged Higgs boson can decay into quarks, while for large $\tan \beta$ they do into leptons. In the last panel of FIG. 2, the total decay width of right-handed neutrinos is given as a function of $\tan \beta$. Because of the seesaw relation in Eq.(3.24), $Y_{\nu}$ is proportional to $\tan \beta$, which cancels $\tan \beta$ dependence in Eqs.(3.29) and (3.30). We found that the lifetime can be as large as $b$ quark, so that secondary vertices may be tagged at the LHC. This property would be the same as $N_{R}$ decay in the $B-L$ model (see, [8]).

In FIG. 4 , we show decay branching fractions of charged Higgs boson, where $m_{H^{ \pm}}=150$ $\mathrm{GeV}$ (first and second) and $200 \mathrm{GeV}$ (third and fourth) are taken. For lower $\tan \beta$ region, charged Higgs bosons mainly decay into quark pairs, while for larger $\tan \beta$ region they do into charged lepton and right-handed neutrino pairs. In large $\tan \beta$ region, main leptonic decay channels are different from the case with $M_{N}>m_{H^{ \pm}}$.

\footnotetext{
$\S$ This prediction would be strongly dependent on the assumption of $R$ and right-handed neutrino mass spectrum. However, we note that something more information about neutrino Yukawa coupling matrix can be obtained by analyzing the charged Higgs decays at colliders.
} 

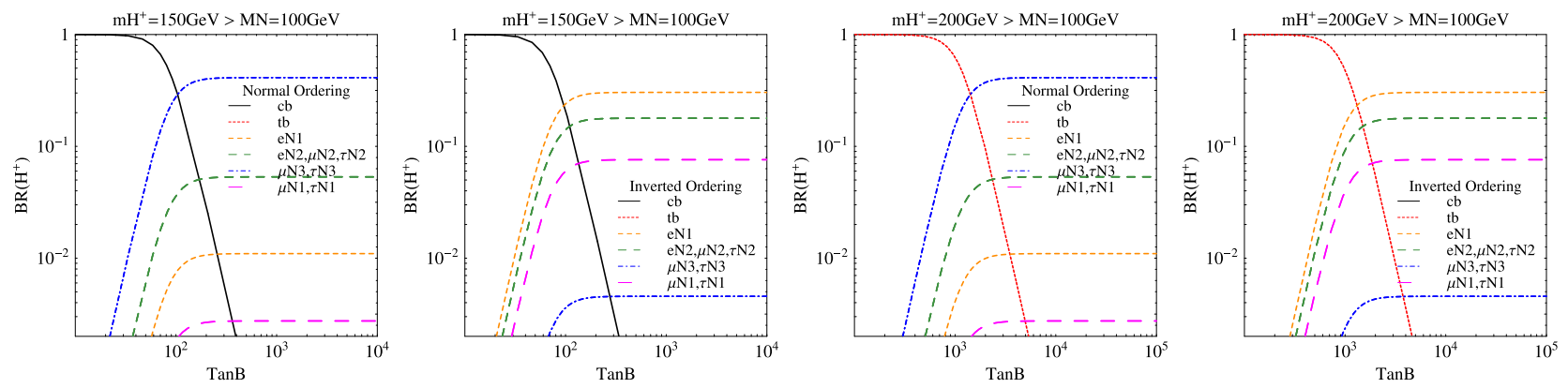

Figure 4: Decay branching ratios of the charged Higgs boson are given for $m_{H^{ \pm}}>M_{N}$, where $R=\mathbf{1}$ and $M_{N} \propto \mathbf{1}$ are taken.
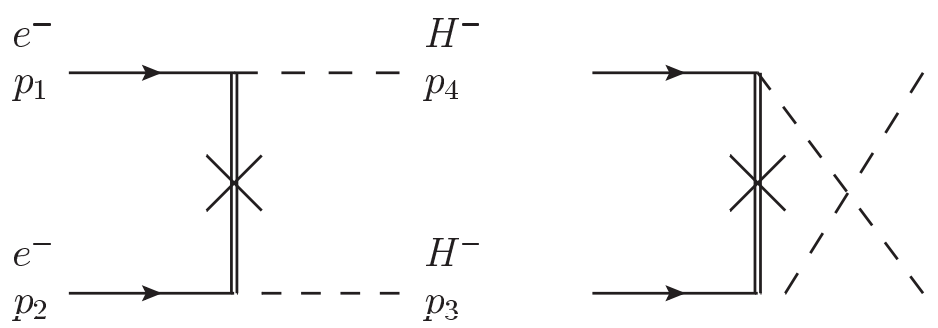

Figure 5: The Feynman diagrams for $e^{-} e^{-} \rightarrow H^{-} H^{-}$in $\nu$ THDM with $N_{R}$.

\subsection{ILC phenomenology}

Here let us investigate some ILC phenomenology. At first we focus on an electron-electron collider, which is a possible option of the ILC. This would be the most important collision process for the $\nu$ THDM to be observed at the ILC. It is because, in the $\nu$ THDM, $e^{-} e^{-}$collision can produce a pair of same sign charged Higgs bosons, where the lepton number violation is occurred in the Majorana mass term of right-handed neutrinos. We stress that the discovery of this lepton number violation is a very important key of the beyond the SM. In FIG. 5, Feynman diagrams for this process are depicted. The differential cross section is calculated as

$$
\frac{d \sigma}{d \cos \theta}=\frac{\beta_{H^{ \pm}}}{256 \pi}\left|Y_{\nu}\left(\frac{M_{N}}{\hat{t}-M_{N}^{2}}+\frac{M_{N}}{\hat{u}-M_{N}^{2}}\right) Y_{\nu}^{T}\right|_{e e}^{2} \simeq \frac{G_{F}^{2} \beta_{H^{ \pm}}}{8 \pi} \tan ^{4} \beta\left|\left\langle M_{\nu}\right\rangle_{e e}\right|^{2}
$$

where $\theta$ is a scattering angle, and $\hat{t}$ and $\hat{u}$ are the Mandelstam variables. The effective mass for the neutrinoless double beta decay is defined as $\left\langle M_{\nu}\right\rangle_{e e}=U_{\mathrm{MNS}} m_{\nu}^{\operatorname{diag}} U_{\mathrm{MNS}}^{T}$. In the last step, we use the seesaw relation and require $\hat{t}, \hat{u} \ll M_{N}^{2}$ to reduce the formula.

Differential cross sections of $e^{-} e^{-} \rightarrow H^{-} H^{-}$with fixed $M_{N}$ are shown in FIGs. 6, where $R=\mathbf{1}$ and $M_{N} \propto \mathbf{1}$ are taken. Collision energies are chosen as $500 \mathrm{GeV}$ and $1000 \mathrm{GeV}$, respectively. Since we take $M_{N} \propto \mathbf{1}$, the normal ordering case and inverted ordering case of the light neutrino mass spectrum give the same distribution. The ratio of VEVs are set to 

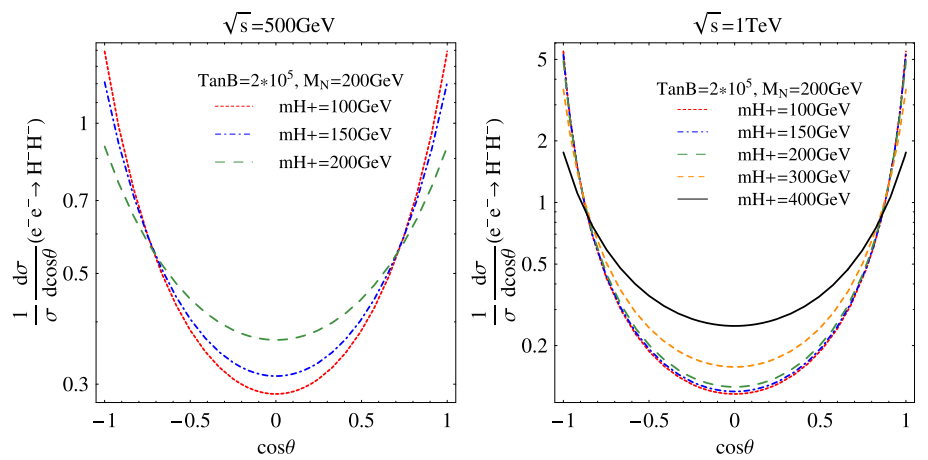

Figure 6: Differential cross sections of $e^{-} e^{-} \rightarrow H^{-} H^{-}$in $\nu$ THDM with $N_{R}$. Mass of righthanded neutrinos is set as $M_{N}=200 \mathrm{GeV}$.
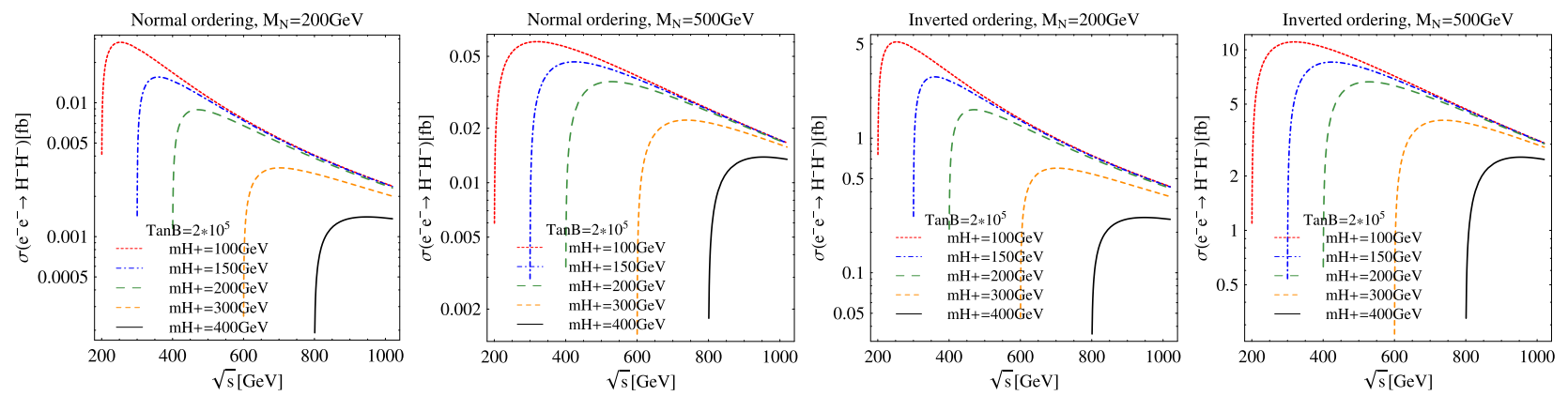

Figure 7: Total cross sections of $e^{-} e^{-} \rightarrow H^{-} H^{-}$in $\nu$ THDM with $N_{R}$. Mass of right-handed neutrinos is set as $M_{N}=200 \mathrm{GeV}$ (first and second) and $500 \mathrm{GeV}$ (third and fourth).

be $\tan \beta=2 \times 10^{5}$, which is allowed by the stringent constraint from $\mu \rightarrow e \gamma$. We can easily see that scattered charged Higgs bosons mainly go to the forward direction. This is a typical behaviour of the co-linear enhancement of the cross section due to the $t(u)$-channel diagram. Performing angle integration, total cross sections are evaluated as a function of the collision energy in FIGs. 7. Masses of right-handed neutrinos are taken to be $200 \mathrm{GeV}$ and $500 \mathrm{GeV}$, respectively. Since we used fixed value of $\tan \beta$, heavier right-handed neutrinos give a larger cross section. Reflecting the neutrino Yukawa matrix, we found that total cross sections can be as large as $0.01 \mathrm{fb}(1 \mathrm{fb})$ for the normal (inverted) ordering of the light neutrino mass spectrum, if $R=1$ and $M_{N} \propto 1$ are satisfied. Polarized beams would be useful to explore the structure of the interaction vertex. Because the vertex is originated from the Yukawa interaction, use of left-handed electrons can enhance cross sections.

In FIG. 8, we show angular distributions of $e^{-} e^{-} \rightarrow H^{-} H^{-}$cross sections for $m_{H^{ \pm}}=$ $150 \mathrm{GeV}$. Masses of right-handed neutrinos are taken as 100,200,300 and $500 \mathrm{GeV}$. As we mentioned before, cross sections become larger for heavier masses of right-handed neutrinos. In FIG. 9, total cross sections are shown as a function of collision energies by changing the mass of right-handed neutrinos. 

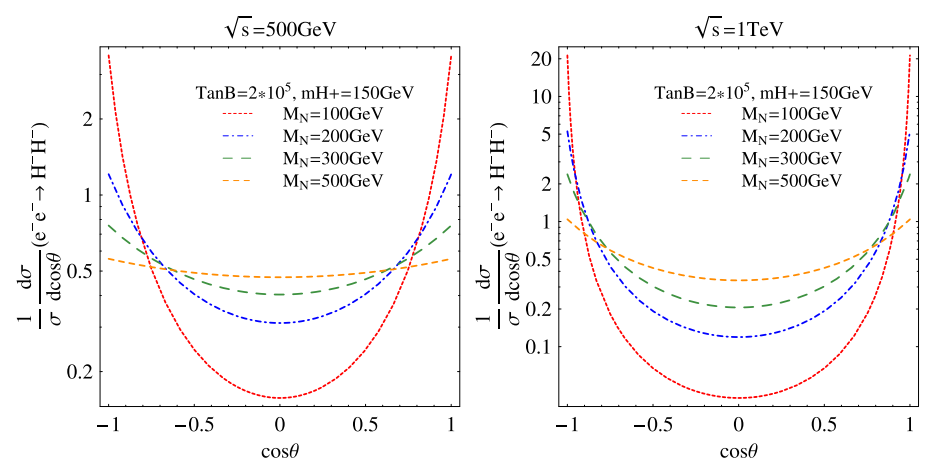

Figure 8: Differential cross sections of $e^{-} e^{-} \rightarrow H^{-} H^{-}$in $\nu$ THDM with $N_{R}$. Mass of charged Higgs bosons is taken to be $m_{H^{ \pm}}=150 \mathrm{GeV}$.
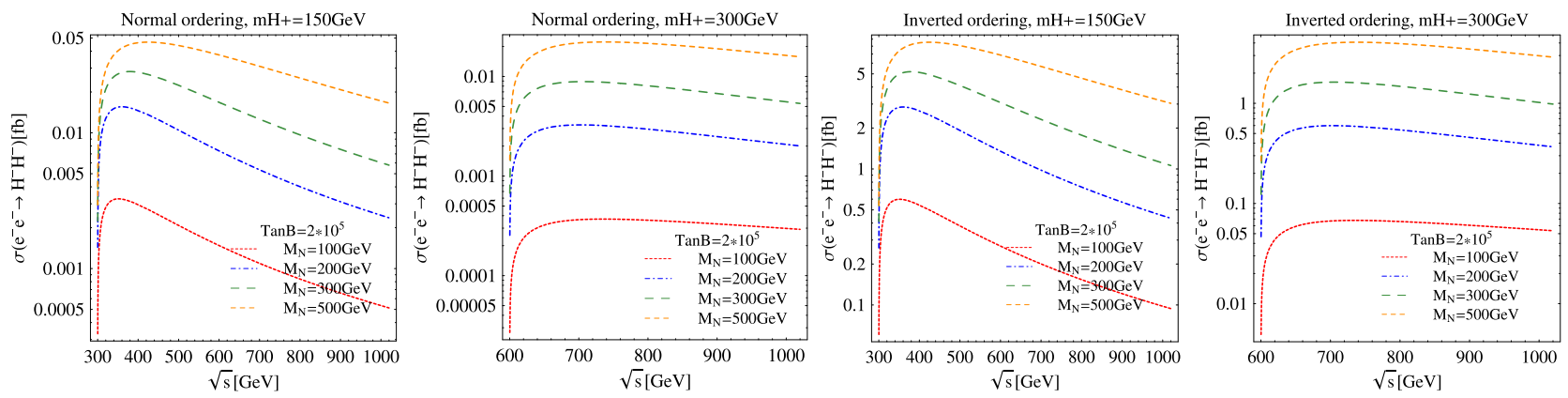

Figure 9: Total cross sections of $e^{-} e^{-} \rightarrow H^{-} H^{-}$in $\nu$ THDM with $N_{R}$. Mass of charged Higgs bosons is taken to be $m_{H}=150 \mathrm{GeV}$ and $300 \mathrm{GeV}$.

Next, we consider the usual $e^{+} e^{-}$collision, the charged Higgs boson pair production via the Drell-Yann process $e^{+} e^{-} \rightarrow H^{+} H^{-}$. Since main decay modes of $H$ and $A$ can be neutrinos, $e^{+} e^{-} \rightarrow A H$ process is also useful as for a source of $N_{R}$. Production cross sections are evaluated in Refs. 27]. In our model, charged Higgs boson can be light, significant number of charged Higgs boson pairs can easily be created, and its specific leptonic decay mode would be detectable. Furthermore, $t$-channel exchange of right-handed neutrinos can also contribute to the process. Because of the chiral structure of the Yukawa interaction, only $\sigma^{L L}$ is changed in the massless limit of electrons, where $L L$ denotes the initial electron and positron polarizations. Differential cross sections are given by

$$
\frac{d \sigma^{L L}}{d \hat{t}}=\frac{d \sigma_{0}^{L L}}{d \hat{t}}\left[1+\left(\frac{1}{8 \pi \alpha} Y_{\nu} \frac{\hat{s}}{\hat{t}-M_{N}} Y_{\nu}^{\dagger}\right)_{e e}\right]^{2},
$$

where $\hat{s}$ and $\hat{t}$ are the Mandelstam variables, and $\sigma_{0}$ is the production cross section of $e^{+} e^{-} \rightarrow$ $H^{+} H^{-}$in the THDM without right-handed neutrinos. The impact of the second term can be one percent level, which may be detectable in the precise measurement of the left-right polarization asymmetry. 
The last is about $e^{-} \gamma$ collision, which is another option of the ILC. Relatively narrow band photons can be generated from electrons and laser beams by the compton back-scattering method. In this option, $e^{-} \gamma \rightarrow H^{-} N$ process may be promising to produce right-handed neutrinos. Production cross sections are not suppressed so strongly because cross sections are only proportional to $Y_{\nu}^{2}[28]$.

Finally, we comment on the production process of right-handed neutrinos at the ILC. Although heavy right-handed neutrinos are gauge singlet, which can interact with electron at tree level via the neutrino Yukawa interaction. The Yukawa couplings can be sizable even under the stringent constraint from the $\mu \rightarrow e \gamma$. Thus, right-handed neutrinos can be produced by the $t$-channel exchange of charged Higgs bosons at ILC as $e^{+} e^{-} \rightarrow N \bar{N}$. The cross sections would be similar to $e^{-} e^{-} \rightarrow H^{-} H^{-}$, because production rates are proportional to $Y_{\nu}^{4}$. Since right-handed neutrinos only couple to the left-handed electron in the massless limit, the production cross section can also be controlled by initial electron and positron polarizations, which may make precision measurement possible.

\section{Summary and discussions}

Smallness of neutrino mass is explained by tiny VEV of extra-Higgs doublet which couples to neutrinos. This model is so-called $\nu$ THDM where TeV-scale seesaw works well, and could be observable at LHC and ILC experiments. Notice that neutrino Yukawa couplings are not tiny in the $\nu$ THDM, which makes the model tested at LHC or ILC. We have investigated collider signatures of the $\nu$ THDM, and shown characteristic signals can be observable at LHC. For example, detective charged tracks can be obtained from long lived charged Higgs when $m_{H^{ \pm}}<M_{N}$. On the other hand, when $m_{H^{ \pm}}>M_{N}$, the charged Higgs bosons mainly decay into a lepton and a right-handed neutrinos. Then right-handed neutrinos can be long-lived similarly to the $B-L$ model, and secondary vertices may be tagged at the LHC. We have also investigated ILC phenomenology such as lepton number violating processes at $e^{-} e^{-}$collision. The discovery of this lepton number violation is a very important key of the beyond the SM.

\section{Acknowledgments}

We thank O. Seto, S. Matsumoto, S. Kanemura, M. Aoki and H. Sugiyama for useful and helpful discussions. This work is partially supported by Scientific Grant by Ministry of Education and Science, Nos. 20540272, 20039006, and 20025004. 


\section{References}

[1] A. Strumia and F. Vissani, arXiv:hep-ph/0606054.

[2] M. Maltoni, T. Schwetz, M.A. Tortola and J.W.F. Valle, New J. Phys. 6 (2004) 122;

G.L. Fogli, E. Lisi, A. Marrone and A. Palazzo, Prog. Part. Nucl. Phys. 57 (2006) 742.

[3] P. Minkowski, Phys. Lett. B 67, 421 (1977); T. Yanagida, in Proceedings of the Workshop on the Baryon Number of the Universe and Unified Theories (KEK, Tsukuba, Japan, 1979), p. 95; M. Gell-Mann, P. Ramond and R. Slansky, in Supergravity, eds. P. van Nieuwenhuizen et al. (North-Holland, 1979), p. 315; R.N. Mohapatra and G. Senjanovic, Phys. Rev. Lett. 44 (1980) 912.

[4] A. Datta, M. Guchait and A. Pilaftsis, Phys. Rev. D 50, 3195 (1994);

F. M. L. Almeida, Y. D. A. Coutinho, J. A. Martins Simoes and M. A. B. do Vale, Phys. Rev. D 62, 075004 (2000);

O. Panella, M. Cannoni, C. Carimalo and Y. N. Srivastava, Phys. Rev. D 65, 035005 (2002);

T. Han and B. Zhang, Phys. Rev. Lett. 97, 171804 (2006);

F. del Aguila, J. A. Aguilar-Saavedra and R. Pittau, JHEP 0710, 047 (2007).

[5] N. Haba, S. Matsumoto and K. Yoshioka, Phys. Lett. B 677, 291 (2009).

[6] X. G. He, S. Oh, J. Tandean and C. C. Wen, Phys. Rev. D 80, 073012 (2009).

[7] K. Huitu, S. Khalil, H. Okada and S. K. Rai, Phys. Rev. Lett. 101, 181802 (2008);

L. Basso, A. Belyaev, S. Moretti and C. H. Shepherd-Themistocleous, Phys. Rev. D 80, 055030 (2009).

[8] L. Basso, A. Belyaev, S. Moretti and C. H. Shepherd-Themistocleous, Phys. Rev. D 80, 055030 (2009).

[9] D. G. Cerdeno and O. Seto, JCAP 0908, 032 (2009).

[10] M. Hashimoto and S. Kanemura, Phys. Rev. D 70, 055006 (2004) [Erratum-ibid. D 70, 119901 (2004)].

[11] E. Ma, Phys. Rev. Lett. 86, 2502 (2001).

E. Ma and M. Raidal, Phys. Rev. Lett. 87, 011802 (2001), [Erratum-ibid. 87, 159901 (2001)].

[12] F. Wang, W. Wang and J. M. Yang, Europhys. Lett. 76, 388 (2006);

S. Gabriel and S. Nandi, Phys. Lett. B 655, 141 (2007). 
[13] E. Ma, Phys. Rev. D 73, 077301 (2006).

[14] S. M. Davidson and H. E. Logan, Phys. Rev. D 80, 095008 (2009).

[15] H. E. Logan and D. MacLennan, Phys. Rev. D 81, 075016 (2010).

[16] N. Haba and M. Hirotsu, Eur. Phys. J. C 69, 481 (2010).

[17] N. Haba and O. Seto, arXiv:1102.2889 [hep-ph].

[18] R. Barbieri, L.J. Hall and V.S. Rychkov, Phys. Rev. D 74, 015007 (2006).

[19] See, for example, J.F. Gunion, H.E. Haber, G.L. Kane, and S. Dawson, The Higgs Hunter's Guide (Westview Press, Boulder, Colorado, 2000).

[20] V. D. Barger, J. L. Hewett and R. J. N. Phillips, Phys. Rev. D 41, 3421 (1990); Y. Grossman, Nucl. Phys. B 426, 355 (1994); M. Aoki, S. Kanemura, K. Tsumura and K. Yagyu, Phys. Rev. D 80, 015017 (2009).

[21] S. Kanemura, Y. Okada, E. Senaha and C. P. Yuan, Phys. Rev. D 70, 115002 (2004).

[22] H. S. Goh, L. J. Hall and P. Kumar, JHEP 0905, 097 (2009).

[23] M.L. Brooks et al. [MEGA Collaboration], Phys. Rev. Lett. 83, 1521 (1999).

[24] J. A. Casas and A. Ibarra, Nucl. Phys. B 618, 171 (2001).

[25] F. T. . Avignone, S. R. Elliott and J. Engel, Rev. Mod. Phys. 80, 481 (2008).

[26] S. S. D. Willenbrock, Phys. Rev. D 35, 173 (1987); O. Brein and W. Hollik, Eur. Phys. J. C 13, 175 (2000); A. A. Barrientos Bendezu and B. A. Kniehl, Phys. Rev. D 64, 035006 (2001).

[27] J. F. Gunion et al., Phys. Rev. D 38, 3444 (1988); A. Djouadi, J. Kalinowski and P. M. Zerwas, Z. Phys. C 57, 569 (1993).

[28] S. Kanemura and K. Tsumura, Phys. Lett. B 674, 295 (2009).

[29] K. Hayasaka, J. Phys. Conf. Ser. 171, 012079 (2009).

[30] O. A. Kiselev [MEG Collaboration], Nucl. Instrum. Meth. A 604, 304 (2009).

[31] J. Prades, Acta Phys. Polon. Supp. 3, 75 (2010).

[32] E. Komatsu et al., arXiv:1001.4538 [astro-ph.CO].

[33] J. L. Feng and M. E. Peskin, Phys. Rev. D 64, 115002 (2001). 